\title{
Comparative Study of Two Flat-Panel X-Ray Detectors Applied to Small-Animal Imaging Cone-Beam Micro-CT
}

\author{
Alejandro Sisniega, Student Member IEEE, Juan José Vaquero, Senior Member IEEE, Eduardo Lage, Student \\ Member IEEE, Álvaro de Carlos, José L. Villena, Mónica Abella, Irina Vidal, Student Member IEEE, \\ Gustavo Tapias, Jose Carlos Antoranz and Manuel Desco
}

\begin{abstract}
This work compares two different X-ray flat-panel detectors for its use in high-speed, cone-beam CT applied to small-animal imaging. The main differences between these two devices are the scintillators and the achievable frame rate. Both devices have been tested in terms of system linearity, sensitivity, resolution, stability and noise properties, taking into account the different timing schemes for each one of them and the mandatory corrections on the raw data. Tomographic scans have been carried out using both detectors to evaluate its final performance as well as the delivered dose needed to achieve similar quality scans. An experimental cone-beam CT test-bench has been designed and implemented to perform the different measurements. It uses a micro-focus $\mathrm{X}$-ray source and a rotating stage where the samples are placed. A modified FDK algorithm has been used to reconstruct the acquired data. Both detectors show similar results for pixel linearity and stability measurements, and their noise levels are comparable. The resolution and sensitivity features are better for the direct grown scintillator detector $(9 \mathrm{lpmm}$ vs. $6 \mathrm{Ipmm}$, and $\sim 4$ times more sensitive for similar delivered dose). Since tomographic reconstructed images for the higher frame-rate detector show acceptable quality, it can be used to implement a faster system for high-speed acquisition techniques like, for example, dynamic imaging or gated protocols.
\end{abstract}

\section{INTRODUCTION}

Cone-beam micro-CT imaging techniques are one of the $\cup_{\text {most }}$ used for small animal imaging studies, by itself or as add-on for functional imaging systems such as PET or SPECT. solving the lack of anatomical information.

Flat-panel type X-ray detector devices are commonly used on this kind of equipment due to their high quality imaging performance and their easy integration. As there are different detector types with different performance, a careful study must

This work is partially funded by the CD-TEAM Project, CENIT Program, Spanish Ministerio de Industria, and with grants from the Ministerio de Educación y Ciencia, projects TEC2007-64731 and TEC2008-06715-C0201.

A. Sisniega, J.J. Vaquero, E. Lage, A de Carlos, J. Villena, M. Abella, I Vidal, G. Tapias and M. Desco are with the Unidad de Medicina y Cirugía Experimental, Hospital General Universitario Gregorio Marañón, Madrid, Spain (J.J. Vaquero email: juanjo@hggm.es)

J. C. Antoranz is with the Departamento de Física, Matemática y Fluidos, Facultad de Ciencias, Universidad Nacional de Educación a Distancia (UNED), Madrid, Spain. be carried out to select the proper device depending on the final application requirements.

Several comparison studies for X-ray detectors have been reported in the previous literature. However, most of them are focused on CCD based detectors and do not take into account the data readout of the different devices [1-3]. There are also some works such as [4-7], which assess the performance of flat-panel detector based tomography systems, measuring the quality of the obtained tomographic data.

In this work, a comparison between two flat-panel X-ray detector models is presented, focusing on their capabilities to be used in high-speed, high-performance micro-CT systems for small-animal imaging.

\section{MATERIALS AND METHODS}

\section{A. Flat-Panel Detectors}

Two flat-panel X-ray semiconductor detectors (C9321SK05 and C7940DK-02, Hamamatsu Photonics K.K.) have been compared. The features reported by the manufacturer for the two devices are shown in Table I.

TABLE I

DETECTOR FEATURES

\begin{tabular}{|l|c|c|}
\hline \multicolumn{1}{|c|}{ Parameter } & C9321SK-05 & C7940DK-02 \\
\hline Pixel size & $50 \mu \mathrm{m}$ & $50 \mu \mathrm{m}$ \\
\hline Number of pixels & $1056 \times 1056$ & $2400 \times 2400$ \\
\hline Frame rate (max) & $27 \mathrm{fr} / \mathrm{sec}$ & $8 \mathrm{fr} / \mathrm{sec}$ \\
\hline Noise (rms) & $1000 \mathrm{elec}$. & $1100 \mathrm{elec}$. \\
\hline Sensitivity & $38 \mathrm{LSB} / \mathrm{mR}$ & $50 \mathrm{LSB} / \mathrm{mR}$ \\
\hline Resolution & $8 \mathrm{lpmm}$ & $10 \mathrm{lpmm}$ \\
\hline Scintillator & $\mathrm{GOS}$ & $\mathrm{CsI}$ \\
\hline
\end{tabular}

The main differences between both systems lie on the scintillator design and on the maximum frame rate.

The C7940DK-02 has a CsI (Cesium lodine) scintillator with a needle structure, grown over the semiconductor surface without any coupling device. This configuration is expected to achieve a high resolution value as well as good sensitivity. 
On the other side, the C9321SK-05 has a GOS (Gadolinium OxiSulfide) scintillator grown over a fiber optic plate flipped on the detector surface. This configuration has an intrinsic poorer resolution, but it achieves a higher frame rate.
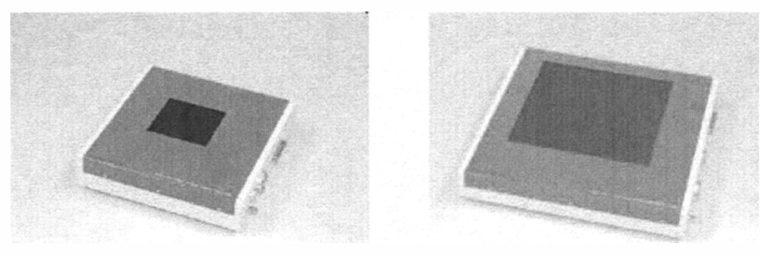

Fig. 1. Hamamatsu flat-panel detectors under test, C9321SK-05 (left) and C7940DK-02 (right).

\section{B. Experimental Setup}

In order to perform the different measurements on the detectors under test, an experimental cone-beam micro-CT has been designed and built.

The system uses a microfocus X-ray source (L9631MOD2, Hamamatsu Photonics K.K) and a stationary rotation stage where the sample is placed. The peak energy achievable by the $\mathrm{X}$-ray source is $110 \mathrm{kV}$, delivering a maximum power of 50 W. A $1 \mathrm{~mm}$ thick aluminum sheet has been used to filter the emitted X-ray beam.

The data acquired by the detector are gathered by a highspeed frame-grabber card (Pixci D2X, Epix inc.). An average Personal Computer (Pentium IV, 2GB RAM) controls the whole system, performing a timing scheme which allows the data acquisition at the highest frame rate offered by the detector devices.

A diagram of the experimental setup is shown Fig. 2. Fig. 3. shows a picture of the developed system.

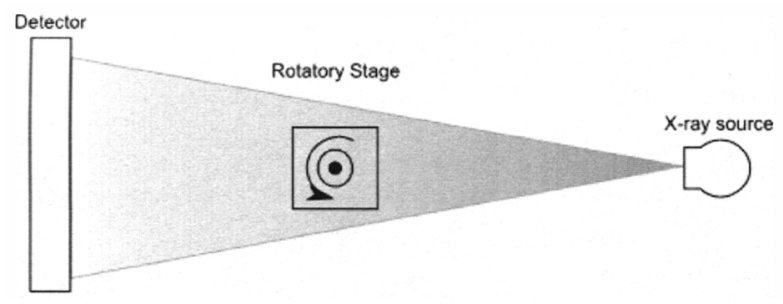

Fig. 2. Diagram of the experimental cone-beam micro-CT

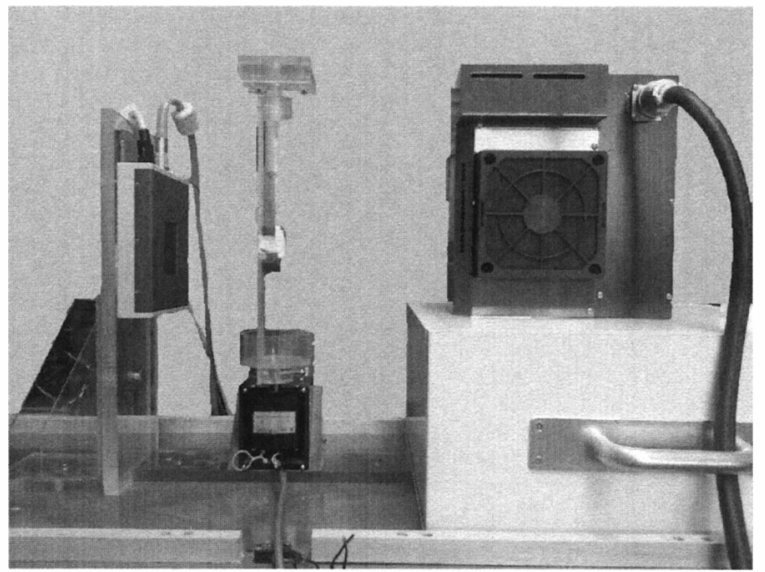

Fig. 3. Experimental cone-beam micro-CT test-bench.

\section{Evaluation Method}

To evaluate the performance of the compared detectors and to validate the use of the C9321SK-05 at its maximum frame rate with high-speed acquisition protocols, we have measured several parameters which are representative of the devices performance.

\section{1) Detector Gain Linearity}

We have measured the system gain linearity as a function of anode current. The output gain level has been estimated as the mean pixel value for each anode current value.

Three different curves have been obtained, using three different X-ray peak energy values, 40, 90 and $110 \mathrm{kV}$.

\section{2) Detector Stability}

To evaluate the temporal stability, the mean pixel value as a function of time for a constant X-ray flux, has been measured.

The test was performed over a total time of 6 hours, taking one sample every minute. The X-ray flux at the detector surface was constant along the whole test time and had $90 \mathrm{kV}$ peak energy.

\section{3) Detector Noise}

To estimate the detector noise, the Signal to Noise Ratio as a function of anode current has been calculated. Three different curves have been obtained, using three different X-ray peak energy values, 40,90 and $110 \mathrm{kV}$.

\section{4) Detector Resolution}

To estimate the device resolution, the planar Modulation to Transfer Function (MTF) has been calculated. The MTF has been obtained using the Nuclear Associates 07-553 test pattern, shown in Fig. 4. 


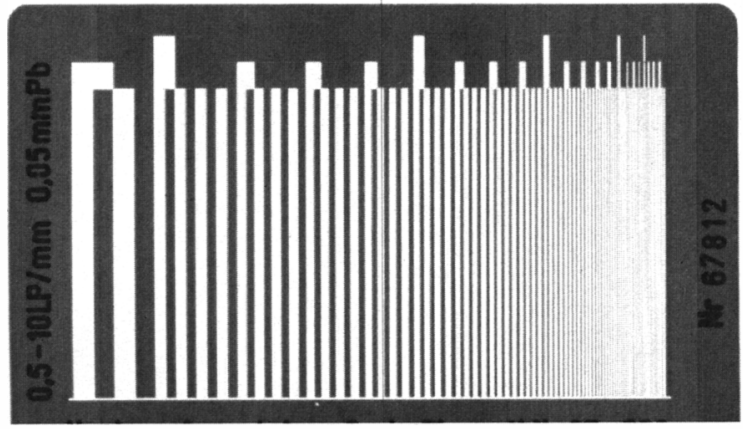

Fig. 4. Nuclear Associates 07-553 test pattern.

5) Relative Detector Sensitivity

To evaluate the difference in the sensitivity achieved by each detector, the ratio of the mean pixel value measured in both detectors has been computed. The datasets have been obtained for equivalent radiation conditions taking into account the different pixel integration time of the detectors.

\section{6) Tomographic Data}

Several tomographic datasets have been acquired using the fastest acquisition protocol allowed by each detector. The different acquisition time has been taken into account to set the anode current of the source.

The projection data have been reconstructed with a modified Feldkamp algorithm, [8].

The quality of the acquired data has been evaluated through the Contrast to Noise Ratio, which was estimated using a phantom with a homogeneous area of nylon and air. The Contrast to Noise Ratio is calculated as:

$$
C N R=\frac{\left|\mu_{n}-\mu_{a}\right|}{\sqrt{\sigma_{n}^{2}+\sigma_{a}^{2}}}
$$

Where $\mu_{\mathrm{n}}$ stands for the mean voxel value in the nylon area, $\mu_{\mathrm{a}}$ for the mean voxel value in the air area and $\sigma_{\mathrm{n}}$ and $\sigma_{\mathrm{a}}$ for the standard deviation of the pixel value inside the nylon and air region, respectively.

To perform the different measurements, the mandatory corrections described in [9] have been applied on the detector raw data.

\section{RESULTS}

Gain linearity measurements are shown in Fig. 5 for C9321SK-05 and in Fig. 6 for C7940DK-02. Both detectors show a linear behavior until they reach their saturation level. The detectors only saturate for the $110 \mathrm{kV}$ peak energy setting.

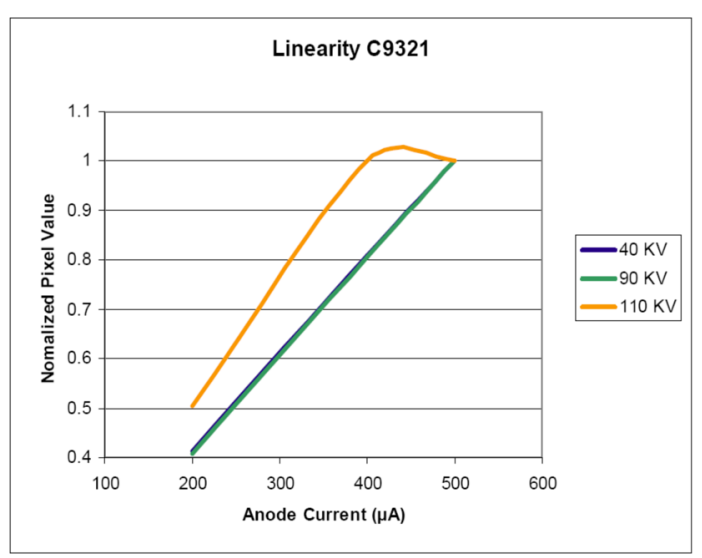

Fig. 5. Gain linearity as a function of anode current for C9321SK-05

The measured temporal stability is plotted in Fig. 7. Both curves yield less than $0.02 \%$ variation in mean pixel value.

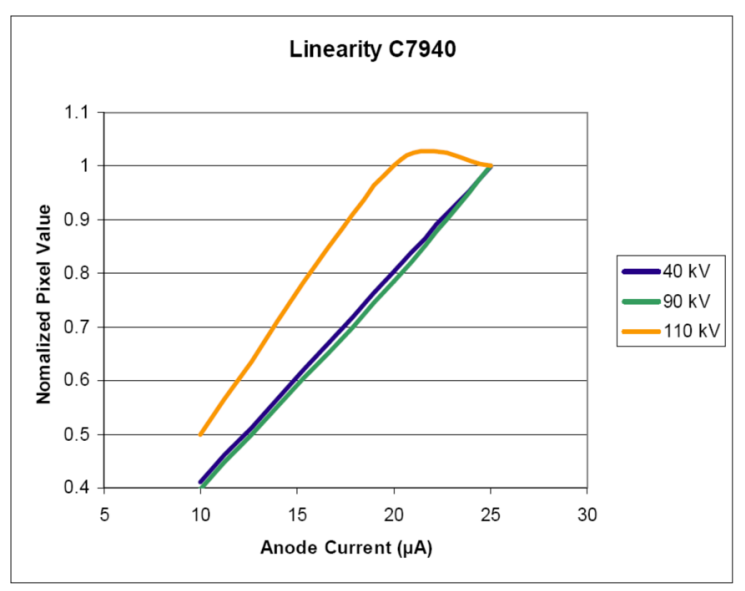

Fig. 6. Gain linearity as a function of anode current for C7940DK-02

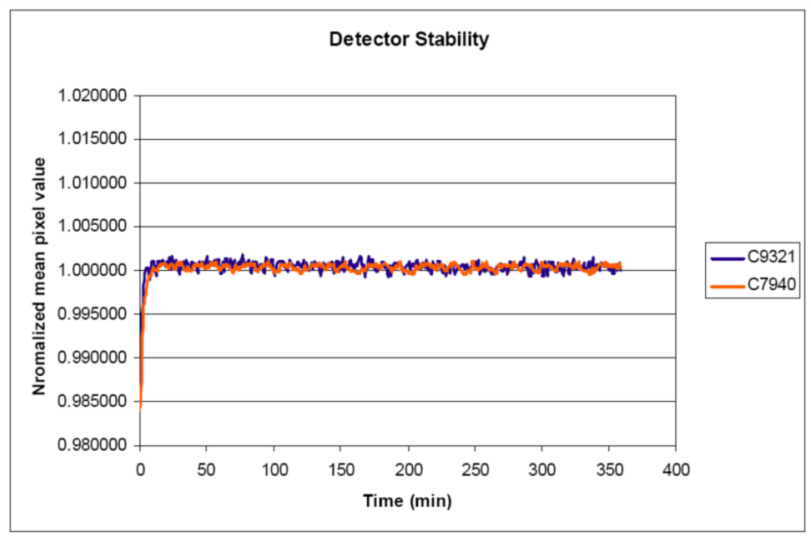

Fig. 7. Time stability for both detectors, estimated as the mean pixel value as a function of time for a constant X-ray flux.

Fig. 8 shows the measured Signal to Noise Ratio as a function of the anode current for the C9321SK-05 detector and 
Fig. 9 for C7940DK-02. The curves meet the expected theoretical value until the detector reaches the saturation level.

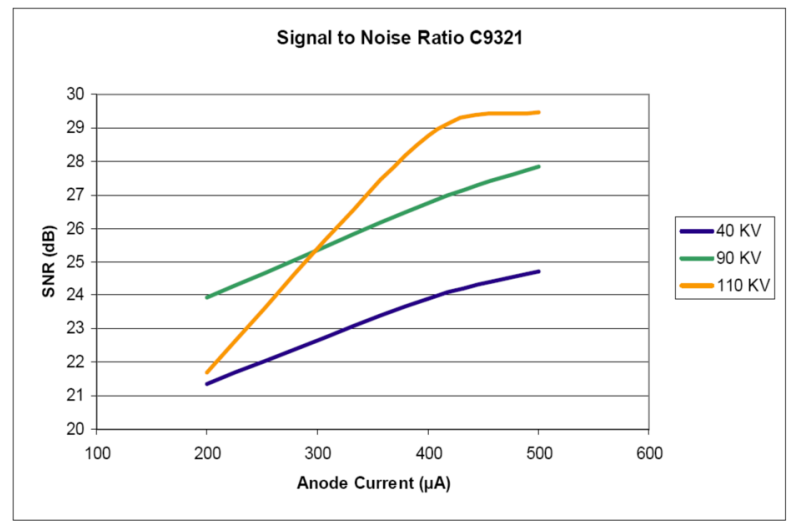

Fig. 8. Signal to Noise Ratio as a function of the source anode current for the C9321SK-05 detector.

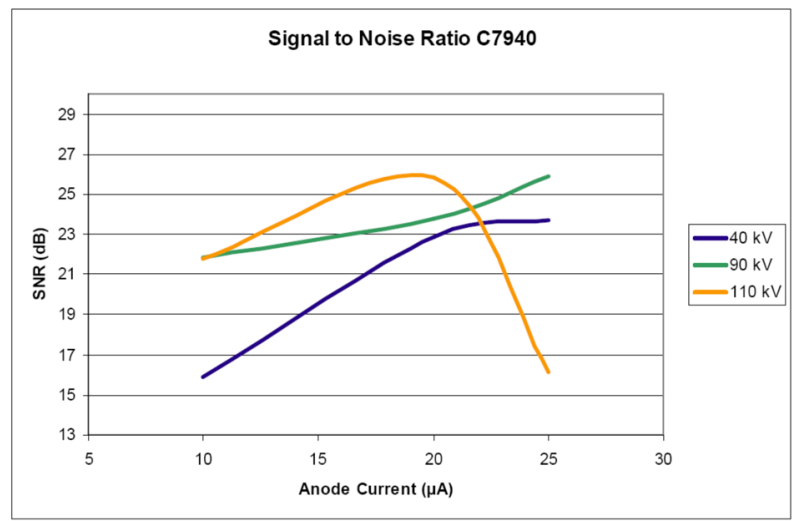

Fig. 9. Signal to Noise Ratio as a function of the source anode current for C7940DK-02

The estimated MTF for the detectors under test can be seen in Fig. 10. The C7940DK-02 shows a better resolution due to the scintillator design. It has a resolution, calculated as the MTF at 5\%, close to $9 \mathrm{lpmm}$, while the C9321SK-05 reaches a value of $6 \mathrm{lpmm}$.

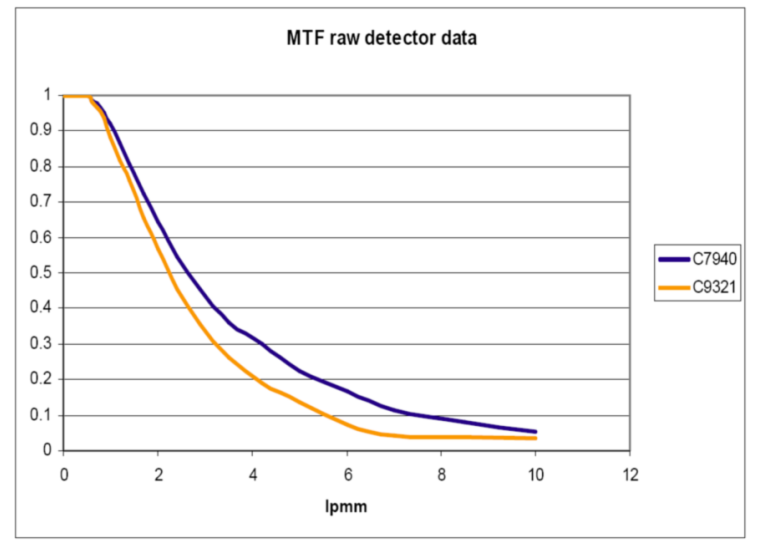

Fig. 10. Estimated MTF
It has been found that the C7940DK-02 is four times more sensitive to the incident radiation than the C9321SK-05.

Regarding the tomographic data, Fig. 11 shows a reconstructed slice of the phantom used to test the quality of the reconstructed images, acquired with the C9321SK-05 using the fastest protocol. The data acquisition took 27 seconds. The X-ray flux had a peak energy of $90 \mathrm{kV}$, with 200 $\mu \mathrm{A}$ anode current.

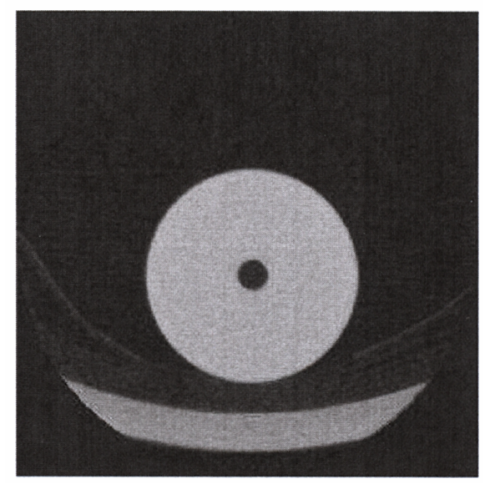

Fig. 11. Reconstructed slice of the test phantom used to estimate the quality of the tomographic data. Acquired with the C9321SK-05 detector using $90 \mathrm{kV}$ X-ray peak energy and $200 \mu \mathrm{A}$ anode current. The total acquisition time was 27 seconds.

Table II gathers the quality measurements performed over some datasets. It can be seen that the use of the C7940DK-02 detector yields a Contrast to Noise Ratio about 4 times greater than the one obtained for the C9321SK-05 detector, delivering approximately the same dose to the sample.

\begin{tabular}{|l|c|c|c|c|}
\cline { 2 - 5 } \multicolumn{1}{c|}{} & $\boldsymbol{T}_{\text {acq }}$ & $\boldsymbol{V}$ & $\boldsymbol{A}_{\text {Anode }}$ & $\boldsymbol{C N R}$ \\
\hline C9321SK-05 & $27 \mathrm{~s}$ & $90 \mathrm{kV}$ & $66 \mu \mathrm{A}$ & 0.82 \\
\cline { 4 - 5 } & & & $200 \mu \mathrm{A}$ & 21.9 \\
\hline C7940DK-02 & $162 \mathrm{~s}$ & $90 \mathrm{kV}$ & $10 \mu \mathrm{A}$ & 3.60 \\
\cline { 4 - 5 } & & & $30 \mu \mathrm{A}$ & 95.4 \\
\hline
\end{tabular}

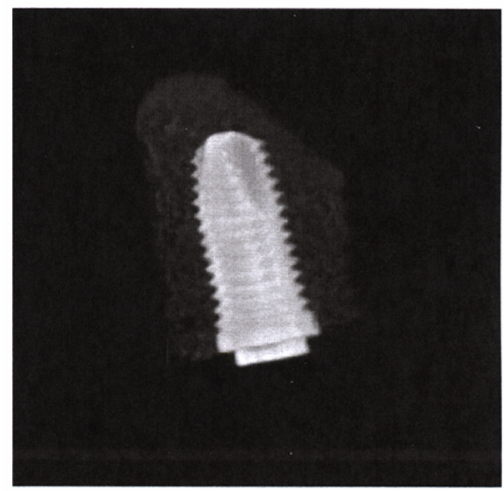

Fig. 12. Volumetric render of a trabecular bone implant acquired using the C9321SK-05 detector. The total acquisition time was 27 seconds for $90 \mathrm{kV}$ $\mathrm{X}$-ray peak energy and $200 \mu \mathrm{A}$ anode current. 


\section{CONCLUSiOnS}

We have compared the performance of two flat-panel X-ray detectors aimed to be used in high speed cone-beam micro-CT systems for small-animal imaging.

The C7940DK-02 which has a CsI needle shaped scintillator, performs better than the C9321SK-05 in terms of sensitivity, resolution and CNR.

Both detectors achieve similar performance in terms of gain linearity, signal-to-noise ratio and stability.

The C9321SK-05 has performed to a level that makes it suitable for high speed acquisition protocols aimed to provide anatomical information complementary to functional data.

\section{ACKNOWLEDGMENT}

The authors thank Hamamatsu Photonics Spain for their support.

\section{REFERENCES}

[1] A. L. Goertzen, V. Nagarkar, R. A. Street, M. J. Paulus, M. J. Boone, and S. R. Cherry, "A comparison of x-ray detectors for mouse CT imaging," Physics in Medicine and Biology, vol. 49, pp. 5251-5265, 2004.

[2] Y. Meng, C. C. Shaw, L. Xinming, C. M. Altunbas, W. Tianpeng, C. Lingyun, T. Shu-Ju, S. C. Kappadath, and C.-J. Lai, "Comparison of two detector systems for cone beam CT small animal imaging - a preliminary study," Proc Soc Photo Opt Instrum Eng., vol. 6142, 2006

[3] T. Gomi, K. Koshida, T. Miyati, J. Miyagawa, and H. Hirano, "An Experimental Comparison of Flat-Panel Detector Performance for Direct and Indirect Systems (Initial Experiences and Physical Evaluation) " Journal of Digital Imaging, vol. 19, pp. 362-370, 2006

[4] C. C. Shaw, C. Lingyun, C. M. Altumbas, T. Shu-Ju, L. Xinming, W Tianpeng, C.-J. Lai, S. C. Kappadath, and Y. Meng, "Cone Beam Brest CT with Flat Panel Detector - Simulation, Implementation and Demonstration," Proceedings of the IEEE, 2005.

[5] J. M. Smyth, D. G. Sutton, and J. G. Houston, "Evaluation of the quality of CT-like images obtained using a commercial flat panel detector system," Biomedical Imaging and Intervention.Journal, vol. 2, 2006

[6] S. C. Thacker, V. V. Nagarkar, H. J. Liang, V. Gaysinskiy, S. Miller, and S. R. Cherry, "Characterization of a novel microCT detector for small animal computed tomogaphy (CT)," Proceedings of SPIE, vol. 6510 , 2007.

[7] J. J. Vaquero, S. Redondo, E. Lage, M. Abella, A. Sisniega, G. Tapıas, M. L. Soto-Montenegro, and M. Desco, "Assessment of a New HighPerformance Small-Animal X-ray Tomograph "IEEE Transactions in nuclear science, vol. In press, 2007.

[8] E. Lage, J. J. Vaquero, S. Redondo, M. Abella, G. Tapias, A. Udias, and M. Desco, "Design and Development of a High Performance Micro-CT System for Small-Animal Imaging," IEEE NSS'M MIC Conference Record. 2006.

[9] Hamamatsu, "X-ray Flat Panel sensor - User's guide." Rev.1.3+ KRII50153. Aug. 2006. 